\title{
Desempenho de diferentes equações antropométricas na predição de gordura corporal excessiva em crianças e adolescentes
}

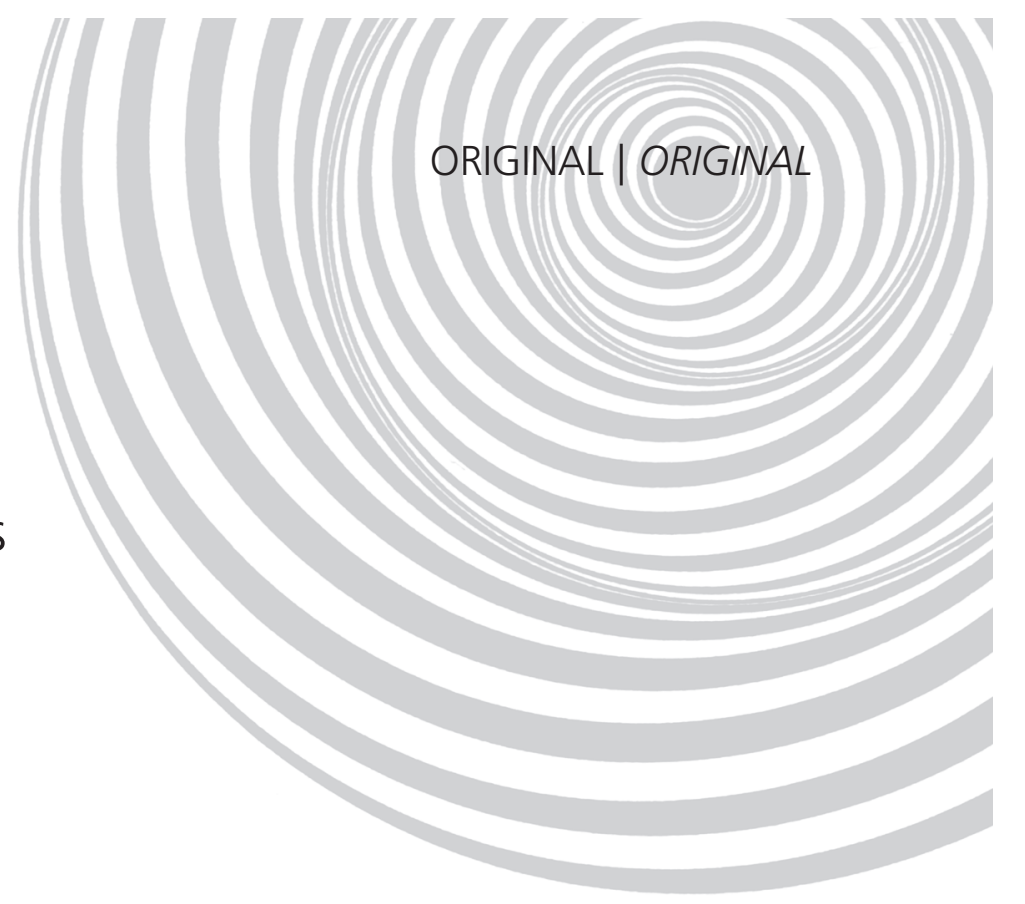

\author{
Performance of different anthropometric \\ equations that predict excess body \\ fat in children and adolescents
}

\author{
Camila BUONANI ${ }^{1}$ \\ Rômulo Araújo FERNANDES² \\ Denise Rodrigues BUENO ${ }^{1}$ \\ Karolynne das Neves BASTOS ${ }^{1}$ \\ Aline Francielle Mota SEGATTO ${ }^{1}$ \\ Loreana Sanches SILVEIRA ${ }^{1}$ \\ Ismael Forte FREITAS JÚNIOR ${ }^{3}$
}

RES U M O

\section{Objetivo}

O objetivo do presente estudo foi analisar a eficiência de diferentes equações antropométricas para a identificação de excesso de gordura corporal entre crianças e adolescentes.

\section{Métodos}

A amostra foi composta por 1498 indivíduos (678 meninos e 820 meninas), com idade entre 7 e 17 anos $(\mathrm{M}=13,7, \mathrm{DP}=2,0)$, da cidade de Presidente Prudente (SP). A massa corporal e a estatura foram mensuradas para o cálculo do Índice de Massa Corporal. Também foi realizada a mensuração da circunferência de cintura e das dobras cutâneas subescapular, tricipital, abdominal e da panturrilha. O percentual de gordura corporal foi calculado por meio de quatro equações: Slaughter et al., Slaughter II et al., Dezenberg et al., e Deurenberg et al.. Para indicar a eficiência das diferentes equações antropométricas na indicação da obesidade, foi utilizada a análise da curva Receiver Operating Characteristic Curve.

\footnotetext{
1 Universidade Estadual Paulista, Programa de Pós-Graduação em Fisioterapia. R. Roberto Simomsen, 305, Bloco III, Centro Universitário, 19060-900, Presidente Prudente, SP, Brasil. Correspondência para/Correspondence to: C. BUONANI. E-mail: <camilabuonani@yahoo.com.br>.

${ }^{2}$ Universidade Estadual Paulista, Programa de Pós-Graduação em Ciências da Motricidade. Rio Claro, SP, Brasil.

3 Universidade Estadual Paulista, Departamento de Educação Física. Presidente Prudente, SP, Brasil.
} 
42 C. BUONANI et al.

\section{Resultados}

No grupo masculino, foram observados valores de sensibilidade elevados (82,4\% a $100,0 \%)$ e valores oscilando de baixo a alto, para a especificidade $(20,9 \%$ a $94,9 \%)$. Para o sexo feminino, os valores de sensibilidade variaram entre moderado e alto $(72,2 \%$ a $99,4 \%)$; e os de especificidade apresentaram, também, grande oscilação entre baixo e alto $(21,6 \%$ a $98,2 \%)$.

\section{Conclusão}

A equação proposta por Deurenberg et al., apresentou o melhor desempenho no diagnóstico tanto da presença como da ausência de obesidade na amostra investigada.

Termos de indexação: Adolescente. Antropometria. Criança. Obesidade.

\section{A B S T R A C T}

\section{Objective}

The purpose of this study was to analyze the efficiency of different anthropometric equations that identify excess body fat in children and adolescents.

\section{Methods}

The sample consisted of 1,498 subjects (678 males and 820 females), aged 7 to 17 years (13.7 SD=2.0), from Presidente Prudente (SP). Body weight and height were measured to calculate body mass index. Waist circumference and triceps, abdominal, and thigh skinfold thicknesses were also measured. The percentage of body fat was estimated by four anthropometric equations: Slaughter et al., Slaughter II et al., Dezenberg et al., and Deurenberg et al.. The Receiver Operating Characteristic Curve was used to indicate the efficiency of these equations.

\section{Results}

High sensitivity values (82.4\% to 100,0\%) and high and low specificity scores (20.9\% to 94.9\%) were observed in males. In females, the sensitivity values ranged from moderate to high (72.2\% to $99.4 \%)$ and the specificity scores were high $(21.6 \%$ to $98.2 \%)$.

\section{Conclusion}

The Deurenberg et al., equation was the most accurate for identifying presence or absence of obesity in this sample.

Indexing terms: Adolescent. Anthropometry. Child. Obesity.

\section{N T R O D U Ç Ã O}

Durante as últimas décadas, tanto em países desenvolvidos como em processo de desenvolvimento, a prevalência de sobrepeso e obesidade entre crianças e adolescentes tem apresentado expressivo crescimento ${ }^{1}$. Suas implicações, tanto em termos individuais como para os sistemas de saúde pública, são alarmantes, uma vez que, já durante a infância e a adolescência, o excesso de peso está associado a diversas morbidades $^{2,3}$, que provocam aumento substancial nos gastos com o seu próprio tratamento e com as suas comorbidades ${ }^{1,4}$.

A estruturação de medidas que sejam eficazes na prevenção e tratamento da obesidade implica, entre outros aspectos, a existência de técnicas que sejam, ao mesmo tempo, simples e eficientes no diagnóstico da doença.

São diversas as técnicas empregadas para estimar a composição corporal total e segmentar, com significativas variações na sua aplicabilidade. De um lado, as técnicas mais sofisticadas, mais precisas e exatas, apresentam custo elevado da avaliação e/ou do equipamento, o que as torna inviáveis em levantamentos populacionais. De outro lado, as técnicas mais simples utilizam equipamentos substancialmente mais baratos e, quando aplicadas com os devidos cuidados com os procedimentos técnicos, são opções viáveis com margens de erro aceitáveis ${ }^{5}$. 
Entre as técnicas mais simples, existem os índices corporais, como o Índice de Massa Corporal (IMC), amplamente usado em estudos populacionais ${ }^{1,6,7}$, que faz a estimativa do estado nutricional baseado nas variáveis massa corporal e estatura. Existem também as equações baseadas em outras medidas antropométricas, como as dobras cutâneas, que podem ser mais eficientes, na medida em que fornecem estimativas mais precisas e exatas da gordura corporal e da massa livre de gordura ${ }^{8}$.

Em razão da sua utilização na estimativa dos componentes da composição corporal (massa de gordura e massa livre de gordura) e, também, pelo fato de que valores elevados de dobras cutâneas estão associados a disfunções fisiológicas, tais como a hipertensão arterial ${ }^{9}$, diversas equações têm sido empregadas no monitoramento do estado nutricional em grandes populações $6,7,10,11$.

Apesar de as equações baseadas em dobras cutâneas apresentarem vantagens em relação às técnicas mais sofisticadas (como Absorptiometria de Raios-X e Dupla Energia, Água Duplamente Marcada e Pesagens Hidrostáticas), com menor custo e maior facilidade de utilização em grupos maiores, ainda assim existe dificuldade em se decidir qual a melhor equação antropométrica. Isso depende da população observada, especialmente quando composta por grupos com características específicas, como, por exemplo, obesos.

No caso da estimativa da composição corporal em indivíduos obesos, especialmente crianças e adolescentes, aumenta a dificuldade quanto àquela decisão, por se tratar de uma população em pleno processo de crescimento, inclusive físico, o que influencia a interpretação e análise dos resultados ${ }^{12}$.

No Brasil, a dificuldade é maior, já que a única equação existente para adolescentes foi elaborada para jovens do sexo masculino, com idade entre 12 e 14 anos $^{11}$, não sendo encontrados estudos que tenham investigado a eficiência de equações antropométricas para a identifi- cação do estado nutricional de crianças e adolescentes brasileiros. Tal fato cria uma limitação importante na literatura, com consequências na utilização dessa técnica na prática cotidiana.

Dessa forma, o objetivo do presente estudo foi analisar a eficiência de diferentes equações antropométricas para a identificação de excesso de gordura corporal entre crianças e adolescentes.

\section{M É T O D O S}

O presente estudo caracteriza-se como descritivo e analítico, de delineamento transversal. Foi realizado na cidade de Presidente Prudente (índice de desenvolvimento humano $[\mathrm{IDH}]=0,846$ ), região oeste do Estado de São Paulo, no ano de 2007. A amostra foi composta por 1498 jovens de ambos os sexos (678 do masculino e 820 do feminino), com idade entre 7 e 17 anos, todos regularmente matriculados em seis escolas das redes pública e particular de ensino (selecionadas aleatoriamente dentre todas as escolas do município e respeitada a proporcionalidade entre escolas públicas e privadas). Os participantes residiam em diferentes regiões da cidade, bem como integravam projetos de extensão (projeto de reeducação alimentar e prática de atividades físicas: $n=193[12,8 \%]$ )/pesquisa (levantamento entre escolares da cidade, abordando a ocorrência de sobrepeso e obesidade: $n=1305$ [87,2\%]), desenvolvidos no campus da Universidade Estadual Paulista (Unesp) em Presidente Prudente. Adotaram-se, como critérios de inclusão: (i) estar devidamente matriculado em alguma escola da rede pública ou privada da cidade; (ii) não apresentar nenhum problema ortopédico que impedisse a avaliação antropométrica; (iii) assinar o termo de consentimento livre e esclarecido.

Aos pais ou responsáveis foi enviado um termo de consentimento livre e esclarecido, onde estavam explícitos o propósito e os métodos empregados no estudo. Foram envolvidos, na amostra final, apenas os jovens que retornaram com o documento devidamente preenchido e assinado. O presente estudo foi aprovado pelo Comitê 
de Ética em Pesquisa da FCT/Unesp (Processo $n^{\circ}$ 087/2006).

A coleta dos dados dos escolares foi realizada nas próprias instituições de ensino, durante o período de aulas, ao passo que os dados dos participantes dos projetos de extensão e pesquisa foram coletados na própria FCT/Unesp, sempre por avaliadores previamente treinados. A idade cronológica dos adolescentes foi determinada de forma centesimal, utilizando-se a diferença entre a data de nascimento e o dia da avaliação.

As variáveis antropométricas analisadas foram massa corporal, estatura, Circunferência de Cintura (CC) e dobras cutâneas. A estatura foi aferida em estadiômetro, com extensão máxima de dois metros e precisão de $0,1 \mathrm{~cm}$. A Massa Corporal (MC) foi mensurada em balança eletrônica, marca Fillizola, com precisão de 0,1 kg e capacidade máxima de $150 \mathrm{~kg}$. Por meio da divisão dos valores de massa corporal pelo valor da estatura elevada ao quadrado, calculou-se o IMC, expresso em $\mathrm{kg} / \mathrm{m}^{2}$.

A mensuração da circunferência de cintura foi efetuada com a utilização de fita métrica metálica, com precisão milimétrica. A mensuração das Dobras Cutâneas Subescapular (DSE), Tricipital (DT), Abdominal (DAb) e da Panturrilha (DPant) foram feitas com adipômetro LANGE (marca Cambridge Scientific Industries), com precisão milimétrica. Todas as medidas antropométricas foram feitas conforme recomendações encontradas na literatura ${ }^{13-15}$.

Para serem considerados com "excesso de gordura", os indivíduos deveriam apresentar, concomitantemente, valores de circunferência de cintura e de IMC superiores aos valores críticos específicos para sexo e idade, conforme proposto por Taylor et al..$^{16}$ e por Cole et al..$^{17}$, respectivamente. Esse procedimento foi utilizado para que fossem minimizados ao máximo os vieses nos resultados da sensibilidade e especificidade.

A estimativa da composição corporal por meio das dobras cutâneas foi feita com a utilização de quatro equações antropométricas:
Equação I - Slaughter et al. ${ }^{18}$. Desenvolvida para crianças brancas e negras 0,61* (DT+DPant) $+5,1$.

Equação II - Slaughter et al. ${ }^{18}$ ( $(35 \mathrm{~mm})$. Desenvolvida para crianças brancas e negras $0,546 *(D T+D S E)+9,7$.

Equação III - Dezenberg et al. ${ }^{19}$. Desenvolvida para crianças brancas e negras, de 4 a 10 anos 0,342 * DT + $(0,256 \cdot M C)+(0,837 \times 1)-7,38$.

Equação IV - Deurenberg et al. ${ }^{20}$. Desenvolvida para crianças com idade $\leq 15$ anos 1,51 * IMC - $(0,70 *$ idade $)-(3,6 * X)+1,4 \times 1=$ masculino e $0=$ feminino.

Tais equações permitiram estimar a gordura corporal em $\mathrm{kg}$ e em percentual (\%GC), e a massa livre de gordura em $\mathrm{kg}$. Para indicar excesso de gordura corporal, foram utilizados os pontos de corte específicos para sexo elaborados por Williams et al. ${ }^{21}$ (sexo masculino: \% GC $\geq 25$; feminino: \%GC $\geq 30$ ).

Quanto a análise estatística, para os dados numéricos, após a utilização do teste de normalidade, foi feita a análise descritiva dos dados. Para os dados categóricos, o teste qui-quadrado $\left(\chi^{2}\right)$ estabeleceu comparações entre as proporções. A correlação de Pearson foi empregada para analisar o relacionamento entre os valores de gordura corporal fornecidos pelas equações com os valores de IMC e CC.

Conforme observado por Sardinha et al. ${ }^{22}$, a simples análise de correlação entre duas variáveis numéricas não é capaz de indicar a eficiência de equações na estimativa de gordura e diagnóstico da obesidade. Segundo os autores, para essa finalidade, devem ser empregados outros procedimentos estatísticos na análise de características de diagnósticos, sendo a Curva Receiver Operating Characteristic Curve (ROC) o mais indicado e aplicado nesses casos. Essa técnica se baseia em três parâmetros: (a) sensibilidade, que representa o verdadeiro positivo, ou seja, indica presença da obesidade por meio das equações antropométricas, quando a mesma também é diagnosticada pelo IMC e CC; (b) especificidade, que representa 
o verdadeiro negativo, ou seja, indica eutrofismo por meio das equações antropométricas, quando o mesmo também é diagnosticado pelo IMC e CC; e (c) área sob a curva (Area Under Curve $A \cup C)$, que representa o coeficiente geral da interação entre sensibilidade e especificidade. Dessa forma, para analisar a eficiência do percentual de gordura (\%GC) indicado pelas equações antropométricas, utilizou-se como referência a indicação simultânea do IMC e da CC, que caracterizaram respectivamente excesso de peso, pelo IMC elevado [IMCe], e excesso de gordura abdominal, pela Circunferência de Cintura elevada [CCe]. A classificação do IMCe e da CCe foi feita pelos pontos de corte propostos por Taylor et al. ${ }^{16}$ e Cole et al. ${ }^{17}$, respectivamente; e as características de diagnóstico foram analisadas por meio da curva ROC e, consequentemente, seus parâmetros (sensibilidade, especificidade e área sob a curva). O tratamento estatístico foi realizado por meio do software Statistical Package for the Social Sciences (SPSS), versão 13.0. A significância estatística foi estabelecida em $5 \%$.

\section{RE S U L T A D O S}

Na Tabela 1, são apresentadas as características gerais da amostra, estratificadas de acordo com o sexo. Com exceção dos valores referentes à idade, as demais variáveis diferiram estatisticamente entre o grupo masculino e o feminino.

A Tabela 2 apresenta a proporção de indivíduos que apresentaram excesso de gordura corporal de acordo com cada uma das equações e, também, do simultâneo excesso de peso e gordura abdominal.

A prevalência de excesso de peso e gordura abdominal, somando-se o IMC e CC, foi diferente, estatisticamente, da prevalência de

Tabela 1. Características gerais de crianças e adolescentes. Presidente Prudente (SP), 2007.

\begin{tabular}{|c|c|c|c|c|c|c|}
\hline \multirow{2}{*}{ Característica } & \multicolumn{2}{|c|}{ Masculino $(n=678)$} & \multicolumn{2}{|c|}{ Feminino $(n=820)$} & \multirow{2}{*}{$t$} & \multirow{2}{*}{$p$} \\
\hline & M & DP & M & DP & & \\
\hline Idade (anos) & 13,70 & 2,20 & 13,80 & 2,30 & $-0,743$ & 0,458 \\
\hline $\mathrm{MC}(\mathrm{kg})$ & 59,10 & 16,70 & 53,80 & 12,20 & 6,700 & 0,001 \\
\hline Estatura (m) & 1,62 & 0,13 & 1,58 & 0,09 & 6,459 & 0,001 \\
\hline IMC $\left(\mathrm{kg} / \mathrm{m}^{2}\right)$ & 22,20 & 5,10 & 21,30 & 4,20 & 3,318 & 0,001 \\
\hline$C C(\mathrm{~cm})$ & 73,90 & 11,70 & 69,50 & 9,50 & 7,977 & 0,001 \\
\hline
\end{tabular}

DP: desvio-padrão; MC: massa corporal; IMC: índice de massa corporal; CC: circunferência da cintura.

Tabela 2. Prevalência (\%) de excesso de gordura corporal pelo IMC e CC e por diferentes equações preditivas, em crianças e adolescentes. Presidente Prudente (SP), 2007.

\begin{tabular}{|c|c|c|c|c|c|}
\hline \multirow{2}{*}{ Equações } & \multirow[b]{2}{*}{ IMC-CC } & \multicolumn{4}{|c|}{ Equações aplicadas no sexo masculino } \\
\hline & & Equação I & Equação II & Equação III & Equação IV \\
\hline Normal & 80,1 & 52,1 & 53,9 & 14,6 & 71,6 \\
\hline Elevada & 19,9 & 47,9 & 46,1 & 85,4 & 28,4 \\
\hline \multirow[t]{3}{*}{$\chi^{2}$ Equação X IMC/CC } & & $p=0,001$ & $p=0,001$ & $p=0,001$ & $p=0,511$ \\
\hline & & \multicolumn{4}{|c|}{ Equações aplicadas no sexo feminino } \\
\hline & IMC-CC & Equação I & Equação II & Equação III & Equação IV \\
\hline Normal & 88,8 & 47,6 & 49,8 & 17,3 & 83,7 \\
\hline Elevada & 11,2 & 52,4 & 50,2 & 82,7 & 16,3 \\
\hline$\chi^{2}$ Equação X IMC/CC & & $p=0,001$ & $p=0,001$ & $p=0,001$ & $p=0,031$ \\
\hline
\end{tabular}

IMC: índice de massa corporal; CC: circunferência de cintura; IMC-CC: prevalência concomitante dos valores de índice de massa corporal e da circunferência da cintura. 
46 C. BUONANI et al.

excesso de gordura corporal fornecida por todas as equações avaliadas, com exceção da equação IV, sendo que todas as equações superestimaram a ocorrência de obesidade indicada, simultaneamente, pelo IMC e CC. Em ambos os sexos, tanto os valores de IMC como os de CC apresentaram relação positiva e significante com todas as equações (dados não apresentados). As Tabelas 3 e 4 apresentam a eficiência das diferentes equações antropométricas no diagnóstico do simultâneo excesso de peso e gordura abdominal em adolescentes do sexo masculino e feminino, respectivamente.

Para o sexo masculino, todas as equações apresentaram valores elevados de sensibilidade

Tabela 3. Eficiência de diferentes equações antropométricas na indicação de elevados valores de gordura corporal entre crianças e adolescentes do sexo masculino. Presidente Prudente (SP), 2007

\begin{tabular}{lrccc}
\hline & \multicolumn{3}{c}{$\begin{array}{c}\text { Simultâneo excesso de peso (IMC) } \\
\text { e gordura abdominal (CC) }\end{array}$} \\
\cline { 2 - 5 } GC $\geq 25 \%$ & SENS & ESP & \multicolumn{3}{c}{ AUC } \\
\cline { 2 - 5 } & & & M & DP \\
\hline Equação I & 97,5 & 73,6 & 0,856 & 0,014 \\
Equação II & 97,5 & 76,1 & 0,868 & 0,014 \\
Equação III & 100,0 & 20,9 & 0,605 & 0,022 \\
Equação IV & 82,4 & 94,9 & 0,886 & 0,017 \\
\hline
\end{tabular}

SENS: sensibilidade; ESP: especificidade; AUC: área sob a curva; IMC: índice de massa corporal; CC: circunferência de cintura; M: média; DP: desvio-padrão.

Tabela 4. Eficiência de diferentes equações antropométricas na indicação de elevados valores de gordura corporal entre crianças e adolescentes do sexo feminino. Presidente Prudente (SP), 2007.

\begin{tabular}{lcccc}
\hline & \multicolumn{3}{c}{$\begin{array}{c}\text { Simultâneo excesso de peso (IMC) } \\
\text { e gordura abdominal (CC) }\end{array}$} \\
\cline { 2 - 5 } GC $\geq 30 \%$ & SENS & ESP & \multicolumn{2}{c}{ AUC } \\
\cline { 4 - 6 } & & & M & DP \\
\hline Equação I & 93,5 & 58,3 & 0,759 & 0,018 \\
Equação II & 95,3 & 61,5 & 0,784 & 0,017 \\
Equação III & 99,4 & 21,6 & 0,605 & 0,022 \\
Equação IV & 72,2 & 98,2 & 0,852 & 0,021 \\
\hline
\end{tabular}

SENS: sensibilidade; ESP: especificidade; AUC: área sob a curva; IMC: índice de massa corporal; CC: circunferência de cintura; M: média; DP: desvio-padrão. (diagnóstico correto da presença do simultâneo excesso de peso e circunferência de cintura elevada [de $82,4 \%$ a $100 \%$ ]) e valores oscilando de baixo a alto para especificidade (diagnóstico correto da ausência do simultâneo excesso de peso e circunferência de cintura elevada [de 20,9\% a 94,9\%]).

Para o sexo feminino, os valores de sensibilidade variaram entre moderado e alto (de $72,2 \%$ a $99,4 \%$ ) e os de especificidade, como no sexo masculino, apresentaram grande oscilação entre baixo e alto (de 21,6\% a 98,2\%).

\section{DIS CUSSÃO}

Neste estudo, a indicação simultânea da obesidade por IMC e obesidade abdominal por CC foram adotados como referências para classificação dos indivíduos em eutróficos ou com excesso de gordura corporal. Esses dois procedimentos apresentam significante relação com a gordura corporal fornecida por meios mais pre$\operatorname{cisos}^{16,23,24}$. Entretanto, dependendo dos pontos de corte adotados, podem variar de maneira significativa na indicação da obesidade ${ }^{25}$. Por essa razão, decidiu-se utilizar os pontos de corte para excesso de peso, tanto do IMC quanto da CC, propostos respectivamente por Taylor et al. ${ }^{16} \mathrm{e}$ Cole et al. ${ }^{17}$, para que não houvesse viés quanto à interpretação dos valores que classificariam os indivíduos em eutróficos e com excesso de gordura corporal.

A utilização desses valores de IMC e de CC (denominados no presente estudo de elevados) tem respaldo nos estudos que revelaram a relação dos mesmos com altos valores de gordura corporal e com disfunções em parâmetros clínicos que afetam a saúde 26,27, especificamente na população pediátrica brasileira ${ }^{25,28}$.

Recentemente, Oliveira ${ }^{29}$ realizou estudo em que se avaliou a eficiência dos pontos de corte baseados no IMC, propostos por Cole et al. ${ }^{17} \mathrm{e}$ Must et al. ${ }^{30}$, por para identificarem sobrepeso e obesidade em adolescentes brasileiros. Os autores concluíram que ambos os critérios são eficientes 
na identificação de adolescentes obesos e eutróficos.

Em outro estudo recente, Guedes \& Rechenchosky ${ }^{31}$ aplicaram o método de Bland \& Altman para avaliar a concordância de quatro equações de estimativa de composição corporal, baseadas em antropometria, realizando estudo com indivíduos entre 18 e 30 anos de idade, Os autores concluíram que, embora os valores das quatro equações sejam similares quando analisados os grupos, os vieses são demasiadamente elevados quando a análise é feita individualmente. Por isso, tais autores recomendam cautela na interpretação dos resultados individuais. Além desses, outros dois estudos recentes chegaram a conclusão semelhante ${ }^{32,33}$, comparando equações preditivas de composição corporal, baseadas em medidas antropométricas, na população adulta brasileira.

Neste estudo, os coeficientes de correlação de Pearson indicaram, para ambos os sexos, escores positivos e significantes entre IMC e CC com o \% GC fornecidos pelas quatro equações antropométricas. Essas correlações estatísticas estão em concordância com outras pesquisas que utilizaram as mesmas medidas ${ }^{26}$ e com grupos de indivíduos de idade semelhante aos do presente estudo ${ }^{23}$.

A técnica estatística de sensibilidade, especificidade e da área sob a curva ROC tem sido tradicionalmente usada para descrever a precisão intrínseca de um teste diagnóstico e também para comparar vários testes diagnósticos ${ }^{34}$.

A utilização da sensibilidade e da especificidade no presente estudo foi feita no sentido de avaliar o grau de eficiência das quatro equações, para indicar os indivíduos que são eutróficos ou com excesso de gordura corporal, tanto pelos valores-ponto de corte de IMCe e CCe quanto pelas equações.

Valores elevados de sensibilidade indicam os verdadeiros positivos, ou seja, aqueles indivíduos que apresentam excesso de gordura tanto pelo IMCe e CCe quanto pela equação, e a especificidade indica os verdadeiros negativos, ou seja, os eutróficos tanto pelo IMCe e CCe quanto pela equação. Nesse sentido, instrumentos extremamente sensíveis e com baixa especificidade identificam com grande eficiência a presença do excesso de peso, mas não são eficientes para indicar os eutróficos.

Quando analisados os dados da sensibilidade, especificidade e área sob a curva ROC, observou-se que, para todas as quatro equações analisadas, eles variaram de moderado a alto, uma vez que o menor valor encontrado foi de $72,2 \%$. Tais resultados indicam que todas as equações discriminam, de maneira satisfatória, tanto os indivíduos eutróficos como aqueles com excesso de gordura corporal.

Apesar desses achados, algumas ressalvas merecem ser discutidas de maneira mais pormenorizada.

As equações desenvolvidas por Slaugther et al. ${ }^{18}$ apresentaram altos valores de sensibilidade (97,5 para o sexo masculino em ambas as equações; e entre 93,5 e 95,3 para o feminino), mas valores não tão elevados especificidade (entre 73,6 e 76, 1 para o sexo masculino; e entre 58,3 e 61,5 para o feminino).

No caso da equação de Dezenberg et al. ${ }^{19}$, os valores de sensibilidade (100 para o sexo masculino e 99,4 para o feminino) foram muito elevados, mas a especificidade foi muito baixa $(20,9$ para o sexo masculino e 21,6 para o feminino).

Esses resultados revelam que as três equações apresentam grandes taxas de casos falso-positivos (indicando a presença da obesidade, quando esta não é diagnosticada pelo método de referência), o que, em termos de sistema de saúde pública, poderia criar um grande viés na interpretação dos resultados, pois indivíduos eutróficos estariam sendo diagnosticados erroneamente como com excesso de gordura.

A equação de Deurenberg et al..20, por sua vez, foi a que apresentou valores mais equilibrados entre os sexos: sensibilidade $(82,4$ para o masculino e 72,2 para o feminino), especificidade 
$(94,9$ e 98,2$)$ e $\operatorname{AUC}(0,886$ e 0,852$)$, respectivamente. Isso indica que, na avaliação do estado nutricional de crianças e adolescentes brasileiros, é ela a equação que apresenta menor viés nos valores estimados em relação aos valores reais, implicando menor erro na interpretação dos resultados.

O fato de o IMCe ter sido utilizado como indicador da obesidade juntamente com a Cce, e também de o próprio IMC estar presente na equação de Deurenberg et al. ${ }^{20}$, poderia ser uma crítica aos resultados obtidos. De fato, se os resultados fossem baseados apenas em análises lineares de correlação, esse ponto seria de grande relevância. Porém, tanto para o IMC como para o \%GC, os pontos de corte adotados foram diferentes, fato que, por si só, já seria um ponto positivo e eliminaria o poder daquela eventual crítica aos resultados. Além disso, com o intuito de esclarecer possíveis dúvidas, uma nova análise foi conduzida com todas as quatro equações, utilizando apenas a CC como referência, e os resultados se mantiveram semelhantes, com a equação de Deurenberg et $a / .^{20}$ apresentando os melhores resultados gerais.

Algumas investigações têm-se preocupado com a validação de técnicas a serem utilizadas na população brasileira, com a adoção de procedimentos mais precisos, visando a validar ou a analisar a concordância das equações antropométricas $^{35}$. Entretanto, não se observam estudos desse tipo com crianças e adolescentes. Dada essa carência e a importância de se adotarem referências e procedimentos de análise confiáveis, o presente estudo apresenta um avanço significativo no conhecimento científico existente na área de análise de técnicas de avaliação do estado nutricional. Não obstante, apesar desses aspectos positivos e de outros, como o tamanho da amostra analisada, o rigoroso controle das variáveis envolvidas, algumas limitações precisam ser apontadas. Nesse sentido, apesar da relativa eficiência do IMC somado à CC na indicação de obesidade em populações pediátricas 25,28 , a principal limitação do presente estudo consistiu em não utilizar métodos mais precisos para a avaliação da composição corporal.

\section{O N C L US Ã O}

Em função dos resultados obtidos, conclui-se que a equação desenvolvida por Deurenberg et al. ${ }^{20}$ mostrou-se mais eficiente que as demais equações antropométricas para o diagnóstico da presença e ausência de excesso de peso e de gordura em crianças e adolescentes brasileiros.

\section{OLABORADORES}

C. BUONANI e I.F. FREITAS JÚNIOR idealizaram o projeto e foram responsáveis pela redação do artigo. R.A. FERNANDES, D.R. BUENO, K.N. BASTOS, A.F.M. SEGATTO e L.S. SILVEIRA participaram das fases finais da redação do artigo.

\section{REFERÊ NCIAS}

1. Wang Y, Monteiro C, Popkin BM. Trends of obesity and underweight in older children and adolescents in the United States, Brazil, China, and Russia. Am J Clin Nutr. 2002; 75(6):971-7.

2. Ferreira AP, Oliveira CE, França NM. Metabolic syndrome and risk factors for cardiovascular disease in obese children: the relationship with insulin resistance (HOMA-IR). J Pediatr (Rio de Janeiro). 2007; 83(1):21-6. doi: 10.2223/JPED.1562.

3. Moreira SR, Ferreira AP, Lima RM, Arsa G, Campbell CS, Simoes $H G$, et al. Predicting insulin resistance in children: anthropometric and metabolic indicators. J Pediatr (Rio de Janeiro). 2008; 84(1): 47-52. doi: 10.2223/JPED.1740.

4. Mokdad AH, Bowman BA, Ford ES, Vinicor F, Marks JS, Koplan JP. The continuing epidemics of obesity and diabetes in the United States. JAMA. 2001; 286(10):1195-200.

5. Heyward VH, Stolarczyk LM. Avaliação da composição corporal aplicada. São Paulo: Manole; 2000.

6. Malina RM, Katzmarzyk PT. Validity of the body mass index as na indicator of the risk and presence of overweight in adolescents. Am J Clin Nutr. 1999; 70(1):131s-6s.

7. Neovius MG, Linné YM, Barkeling BS, Rossner SO. Sensitivity and specificity of classification systems of fatness in adolescents. Am J Clin Nutr. 2004; 80(3):597-603.

8. Lohman TG, Caballero B, Himes JH, Davis CE, Stewart D, Houtkooper L, et al. Estimation of body 
fat from anthropometry and bioelectrical impedance in Native American children. Int J Obes Relat Metab Disord. 2000; 24(8):982-8.

9. Araújo TL, Lopes MV, Cavalcante TF, Guedes NG, Moreira RP, Chaves ES, et al. Análise de indicadores de risco para hipertensão arterial em crianças e adolescentes. Rev Esc Enferm USP. 2008; 42(1):120-6. doi: 10.1590/S0080-62342008000100016.

10. Horlick M, Arpadi SM, Bethel J, Wang J, Moye J Jr, Cuff $P$, et al. Bioelectrical impedance analysis models for prediction of total body water and fat-free mass in healthy and HIV-infected children and adolescents. Am J Clin Nutr. 2002; 76(5):991-9.

11. Salem M, Pires Neto CS, Waissmann W. Equações nacionais para a estimativa da gordura corporal de brasileiros. Rev Educ Física. 2007; 136(1):66-78.

12. GuedeS DP, Guedes, JERP. Recursos utilizados no estudo do crescimento e da composição corporal. In: GuedeS DP, Guedes JERP. Crescimento, composição corporal e desempenho motor. São Paulo: CLR Balieiro; 1997. p.50-3.

13. Gordon CC, Chumlea WC, Roche AF. Stature, recumbent length and weight. In: Lohman TG, Roche AF, Martorell $R$, editors. Anthropometric standardization reference manual. Champaign (IL): Human Kinetics; 1988. p.3-8.

14. Harrison GG, Buskirk ER, Carter JEL, Johnston FE, Lohman TG, Pollock ML, et al. Skinfold thicknesses and measurement technique. In: Lohman TG, Roche AF, Martorell R, editors. Anthropometric standardization reference manual. Champaign (IL): Human Kinetics; 1988. p.55-70.

15. Callaway CW, Chumlea WC, Bouchard C, Himes $\mathrm{JH}$, Lohman TG, Martin AD, et al. Circunferences. In: Lohman TG, Roche AF, Martorell R, editors. Anthropometric standardization reference manual. Champaign (IL): Human Kinetics; 1988. p.39-54.

16. Taylor RW, Jones IE, Williams SM, Goulding A. Evaluation of waist circumference, waist-to-hip ratio, and the conicity index as screening tools for high trunk fat mass, as measured by dual-energy X-ray absorptiometry, in children aged 3-19 y. Am J Clin Nutr. 2000; 72(2):490-5.

17. Cole TJ, Bellizzi MC, Flegal KM, Dietz WH. Establishing a standard definition for child overweight and obesity worldwide: international survey. BMJ. 2000; 320(7244):1240-3.

18. Slaughter, MH, Lohman TG, Boileau RA, Horswill CA, Stillman RJ, Van Loan MD, et al. Skinfold equations for estimation of body fatness in children and youth. Hum Biol. 1988; 6(5):709-23.

19. Dezenberg CV, Nagy TR, Gower BA, Johnson R, Goran Ml. Predicting body composition from anthropometry in pre-adolescent children. Int J Obes Relat Metab Disord. 1999; 23(3):253-9.
20. Deurenberg P, Weststrate JA, Seidell JC. Body mass index as a measure of body fatness: age- and sex-specific prediction formulas. Br J Nutr. 1991; 65(2):105-14.

21. Williams DP, Going SB, Lohman TG, Harsha DW, Srinivasan SR, Webber LS, et al. Body fatness and risk for elevated blood pressure, total cholesterol, and serum lipoprotein ratios in children and adolescents. Am J Public Health. 1992; 82(3):358-63.

22. Sardinha LB, Going SB, Teixeira PJ, Lohman TG. Receiver operating characteristic analysis of body mass index, triceps skinfold thickness, and arm girth for obesity screening in children and adolescents. Am J Clin Nutr. 1999; 70(6):1090-5.

23. Eto C, Komiya S, Nakao T, Kikkawa K. Validity of the body mass index and fat mass index as an indicator of obesity in children aged 3-5 year. J Physiol Anthropol Appl Human Sci. 2004; 23(1): 25-30.

24. Mei Z, Grummer-Strawn LM, Pietrobelli A, Goulding A, Goran MI, Dietz WH. Validity of body mass index compared with other body-composition screening indexes for the assessment of body fatness in children and adolescents. Am J Clin Nutr. 2002; 75(6): 978-85.

25. Fernandes RA, Rosa CSC, Buonani C, Bueno DR, Oliveira AR, Freitas Júnior IF. Desempenho de diferentes valores críticos de índice de massa corporal na identificação de excesso de gordura corporal e obesidade abdominal em adolescentes. Rev Assoc Med Bras. 2007; 53(6):515-9. doi:10.1590/S010 4-42302007000600019.

26. Flegal KM, Shepherd JA, Looker AC, Graubard BI, Borrud LG, Ogden CL, et al. Comparisons of percentage body fat, body mass index, waist circumference, and waist-stature ratio in adults. Am J Clin Nutr. 2009; 89(2):500-8. doi: 10.3945/ajcn. 2008.26847.

27. Christian AH, Mochari H, Mosca LJ. Waist circumference, body mass index, and their association with cardiometabolic and global risk. J Cardiometab Syndr. 2009; 4(1):12-9. doi:10.1111/ j.1559-4572.2008.00029.x.

28. Almeida CA, Pinho AP, Ricco RG, Elias CP. Abdominal circumference as an indicator of clinical and laboratory parameters associated with obesity in children and adolescents: comparison between two reference tables. J Pediatr (Rio de Janeiro). 2007; 83(2):181-5. doi:10.2223/JPED.1604.

29. Oliveira FL, Taddei JA, Escrivao MA, Cobayashi F, Barros ME, Vitolo MR, et al. Accuracy of obesity diagnosis in Brazilian adolescents: comparison of Cole et al and Must et al criteria with DXA percentage of fat mass. Nutr Hosp. 2006: 21(4):484-90. 
30. Must A, Dallal GE, Dietz WH. Reference data for obesity: 85th and 95th percentiles of body mass index (wt/ht2) and triceps skinfold thickness. Am J Clin Nutr. 1991; 53(4):839-46.

31. Guedes DP, Rechenchosky L. Comparação da gordura corporal predita por métodos antropométricos: índice de massa corporal e espessuras de dobras cutâneas. Rev Bras Cineantropom Desempenho Hum. 2008: 10(1):1-7.

32. Salem M, Fernandes Filho J, Pires Neto CS. Desenvolvimento e validacão de equacões antropométricas específicas para a determinacão da densidade corporal de mulheres militares do Exército Brasileiro. Rev Bras Med Esporte. 2004:10(3):141-51.

33. Rech CR, Santos DP, Silva JCN. Desenvolvimento e validação de equações antropométricas para pre- dição da gordura corporal em mulheres entre 50 e 75 anos de idade. Rev Bras Cineantropom Desempenho Hum. 2006; 8(1):5-13.

34. Hanley JA, McNeil BJ, The meaning and use of the area under a receiver operating characteristic (ROC) curve. Radiology. 1982; 143(1):29-36.

35. Rodrigues MN, Silva SC, Monteiro WD, Farinatti PTV. Estimativa da gordura corporal através de equipamentos de bioimpedância, dobras cutâneas e pesagem hidrostática. Rev Bras Med Esporte. 2001; 7(4):125-31.

Recebido em: 9/1/2009

Versão final reapresentada em: 5/8/2010 Aprovado em: 22/9/2010 